\title{
Análise do comportamento de avaliação de marcas: nível de reforço informativo programado e ponto de venda
}

\author{
Analysis of brand evaluation behavior: programmed informational \\ reinforcement level and point of sale \\ Análisis del comportamiento de evaluación de marca: información \\ programada nivel de impulsor y punto de venta
}

\section{Daiane Kachuba ${ }^{1}$, Jorge M.Oliveira-Castro²}

[1] [2] Universidade de Brasília (UNB) I Título abreviado: Análise do comportamento de avaliação de marcas | Endereço para correspondência: Endereço físico para correspondência: Universidade de Brasília, Instituto de Psicologia, Departamento de Processos Psicológicos Básicos. Instituto de Psicologia - Campus Universitário Darcy Ribeiro. Asa Norte, Brasília - DF, Brasil. CEP: 70910-900. I Email: daianekachuba15@gmail.com I doi: doi.org/10.18761/PAC.2020.v11.n1.03 


\begin{abstract}
Behavioral Analysis Principles have been applied to interpret and explain consumption-related phenomena through the Behavioral Perspective Model (BPM). The BPM makes it possible to interpret part of the phenomena that marketing calls brand differentiation as programmed informational reinforcement, typically measured by the Mean Knowledge and Quality (MKQ). Considering that one of the important variables of a brand's context is where it is sold to the final consumer, the main objective of the research was to examine the relationship between brand differentiation and point of sale. The data were collected through a questionnaire, answered by 120 people, which contained brands of women's fashion stores, with points of sale in three qualitatively different shopping malls in Brasília, frequented by different groups. Results indicated that the same brand signals different levels of informational reinforcement depending on the point of sale, and higher informational malls increase the potential social reinforcements associated with the brands. In addition, the paper demonstrates the usefulness of behavior-analytic theory in examining brand-choice behaviors by using MKQ as a proximal scale to measure informational reinforcements as programmed social contingencies.
\end{abstract}

Keywords: brands differentiation, point-of-sale, Behavioral Perspective Model, informational reinforcement, consumer behavior.

Resumen: Los principios del análisis conductual se han aplicado para interpretar y explicar los fenómenos relacionados con el consumo, a través del Behavioral Perspective Model (BPM). BPM permite interpretar como refuerzo informativo programado, típicamente medido por el promedio de conocimiento y calidad, parte de los fenómenos que el marketing llama de diferenciación de la marca. Considerando que una de las variables importantes del contexto de una marca es el lugar donde se vende al consumidor final, el objetivo principal de la investigación fue examinar la relación entre la diferenciación de la marca y el punto de venta. Los datos se recopilaron a través de un cuestionario, respondido por 120 personas, que contenía marcas de tiendas de moda femenina, con puntos de venta en tres centros comerciales cualitativamente diferentes en Brasilia, frecuentados por diferentes grupos. Los resultados indicaron que la misma marca señala diferentes niveles de refuerzo informativo según el punto de venta, y los centros comerciales informativos más altos aumentan los posibles refuerzos sociales asociados con las marcas. Además, el documento demuestra la utilidad de la teoría analítica del comportamiento en el examen de los comportamientos de elección de marca mediante el uso de promedio de conocimiento y calidad como una medida proximal para medir los refuerzos informativos como contingencias sociales programadas.

Palabras clave: diferenciación de marca, punto de venta, Behavioral Perspective Model, refuerzo informativo, comportamiento del consumidor. 
De acordo com as teorias e práticas predominantes na área de marketing, estratégias de segmentação de mercado e de diferenciação de produtos objetivam atender à diversidade de demanda dos consumidores, que diferem quanto às suas necessidades e preferências (Kotler \& Keller, 2012; Smith, 1956; Wedel \& Kamakura, 2012). Geralmente, os três tipos principais de segmentação de mercado se baseiam em: atributos físicos - dados demográficos, geográficos e socioeconômicos; atributos psicológicos gerais - análises do estilo de vida do consumidor; e especificidades do produto - benefícios dentro da categoria (Kotler \& Keller, 2012; Vyncke, 2002; Wells, Chang, Oliveira-Castro, \& Pallister, 2010). Em cada segmento, os ofertantes procurariam diferenciar seu produto com o objetivo de interferir na demanda, proteger o negócio da afluência de preços e obter vantagem competitiva. Isso tipicamente é feito por intermédio da publicidade ou da introdução de aspectos diferenciais em relação aos produtos dos concorrentes, como qualidade, estilo ou imagem (Kotler \& Keller, 2012). Ao diferenciar o produto, fabricantes procuram aumentar as vendas mesmo que os preços estejam acima dos níveis de equilíbrio de mercado perfeitamente competitivo (Smith, 1956). A partir do momento em que todas as possibilidades de diferenciar o produto são atendidas, os ofertantes passam a adotar estratégias para se diferenciar dos concorrentes por meio de sua marca.

De acordo com a literatura de marketing, marcas desempenham diversas funções, tais como de simplificar a escolha e indicar qualidade para os consumidores, identificar uma empresa em meio a diversidade de ofertas, refletir a eficácia da propaganda, além de ser um ativo financeiro (Keller \& Lehmann, 2006). Marcas de luxo, por exemplo, podem se tornar líderes em seu campo, ter sua excelência reconhecida, movimentar grande volume de dinheiro e gerar emprego e renda. Isso pode ocorrer tanto na comercialização quanto na produção dos bens, além de também poderem estabelecer preços acima dos níveis de equilíbrio de mercado (Doyle \& Moore, 2018). Esses são alguns dos objetivos típicos das estratégias de diferenciação de marcas, as quais, de acordo com alguns autores (e.g., Aaker, 1997; Austin, Siguaw, \& Mattila, 2003), podem se diferenciar ao ponto de desenvolver características de personalidade. Encontra-se implícita nesse tipo de metáfora a concepção de que as características de uma marca permanecem relativamente estáveis em diferentes momentos e contextos, de maneira semelhante ao que ocorre com as pessoas.

Esse tipo de teorização ilustra a forte predominância na área de comportamento do consumidor e marketing, nas últimas décadas, de teorias psicológicas de inspiração social cognitiva. De acordo com tais teorias, o comportamento do consumidor tem sido explicado com base em construtos psicológicos, que seriam eventos mentais, tais como atitudes, valores, expectativas, satisfação e intenção, que causam o comportamento resultante do consumidor. A ênfase predominante e quase exclusiva se dá em variáveis intra-individuais, com pouco foco nas variáveis situacionais que possam influenciar o comportamento (cf. Foxall, 1997).

Essa mesma ênfase tem prevalecido na área de marketing quando se procura explicar o "comportamento" de marcas e produtos. Muitas explicações têm sido formuladas com base no arcabouço teórico-conceitual da Psicologia Social Cognitiva. Em decorrência disso, observa-se a utilização de concepções metafóricas de acordo com as quais marcas teriam, por exemplo, atitudes, personalidade e valores (e.g., honesta).

Com o objetivo de preencher essa lacuna e fornecer explicações consistentes com uma teoria sobre comportamento, princípios comportamentais têm sido aplicados para interpretar e explicar os fenômenos relacionados ao consumo, fundamentados nos conceitos da Análise do Comportamento e Economia Comportamental (cf. Foxall, 1990, 1998, 2017). Essa abordagem comportamental enfatiza a influência das variáveis situacionais sobre o comportamento, pois adota uma teoria selecionista, de acordo com a qual o comportamento é selecionado pelas consequências que produzem no ambiente (Baum, 2006).

\section{Interpretação comportamental de diferenciação de marcas}

O principal arcabouço teórico adotado na Análise do Comportamento do Consumidor tem sido o Behavioral Perspective Model (BPM). Ele foi desen- 
volvido com base no modelo de seleção por consequências de Skinner (e.g., 1953, 1957) para explicar o comportamento do consumidor baseado em eventos antecedentes e consequentes em relação à situação de consumo. O comportamento do consumidor ocorre na interseção entre o cenário de consumo e a história de aprendizagem do indivíduo, denominada situação de consumo, interseção esta que prevê o comportamento do consumidor (Foxall, 1998, 2010, 2017; Oliveira-Castro \& Foxall, 2015; Oliveira-Castro et al., 2008).

O cenário de consumo é definido como o ambiente físico, social e temporal, no qual o consumidor é exposto a eventos, que funcionam como estímulos discriminativos, sinalizando as consequências para as possíveis escolhas e, com isso, fazendo com que seu comportamento varie. O grau de controle do ambiente sobre o comportamento depende do nível de abertura do cenário. Um ambiente mais aberto permite que o consumidor se comporte de forma relativamente livre, pois apre- senta diversas alternativas de escolha e possibilidades de respostas (e.g., assistir presencialmente a um show de rock). Em um ambiente mais fechado, o consumidor será menos livre para fazer escolhas, no sentido de ser exposto a um menor número de alternativas e possibilidades de respostas, e estará ajustado a um padrão de comportamento definido por outra pessoa, muitas vezes com a presença de algum controle punitivo (e.g., assistir presencialmente a uma ópera) (Oliveira-Castro \& Foxall, 2015; Oliveira-Castro et al., 2008).

Experiências semelhantes que o consumidor teve anteriormente (i.e., história de aprendizagem individual), relacionam-se com os elementos do cenário para prever as consequências comportamentais. Assim, os eventos antecedentes ao comportamento sinalizam a possibilidade de quatro tipos de consequências: reforço utilitário, reforço informativo, punição utilitária e punição informativa (Oliveira-Castro \& Foxall, 2015; Oliveira-Castro et al., 2008), conforme apresentado na Figura 1.

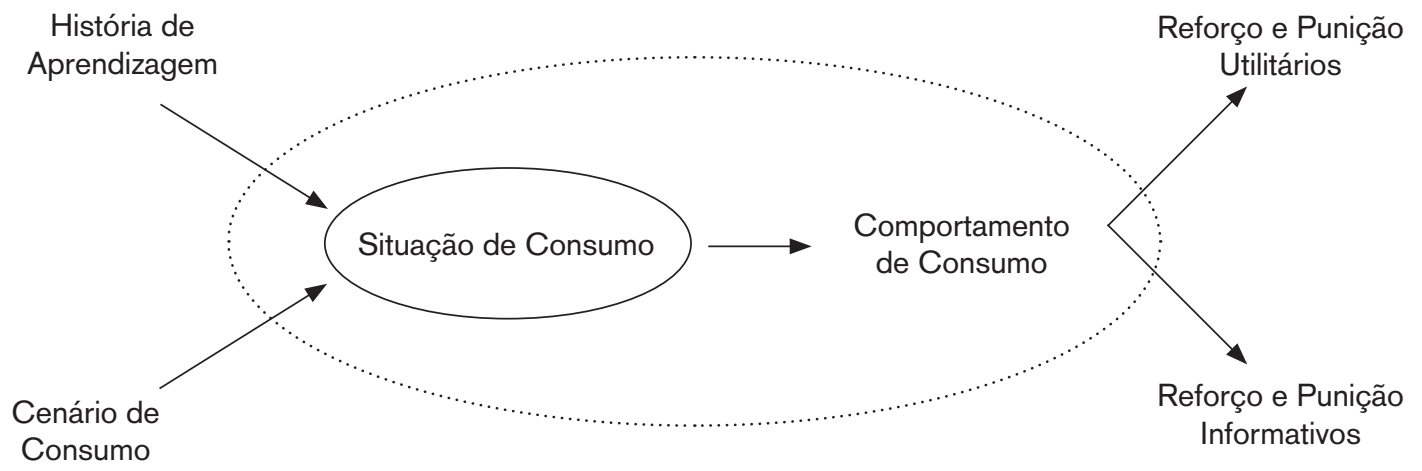

Figura 1: Esquema do Behavioral Perspective Model (BPM). Adaptado de Foxall (2017).

O reforço utilitário consiste em benefícios funcionais e se refere às consequências associadas ao aumento da utilidade para o indivíduo, mediadas e obtidas a partir da compra ou uso do produto ou serviço. Por exemplo, tipicamente, o principal reforço utilitário para adquirir um automóvel é obter transporte de porta a porta. O reforço informativo, por outro lado, é social, mediado pelas ações e reações de outras pessoas e associado ao feedback sobre o desempenho do consumidor, in- dicando o nível de adequação do comportamento. Ou seja, o reforço informativo geralmente é derivado do nível de status social e prestígio que o consumidor obtém quando compra ou usa determinado bem. No exemplo do automóvel, reforço informativo pode advir de comentários e reações elogiosas das pessoas associadas à compra de uma marca ou modelo de automóvel com alto nível de prestígio (e.g., BMW, Audi) (Oliveira-Castro \& Foxall, 2015). 
A punição utilitária se relaciona ao dinheiro gasto, ao tempo despendido na procura ou às consequências aversivas funcionais, como comprar um produto danificado. Já a punição informativa ocorre quando outras pessoas não aprovam ou criticam o que o consumidor comprou, ou, ainda, quando o consumidor descobre que pagou muito pelo produto. De forma geral, tanto reforços quanto punições utilitárias e informativas estão presentes em todos os produtos, variando em cada um e em distintas situações (Oliveira-Castro et al., 2008). Marcas também fornecem certa magnitude dos dois tipos de reforços. Desse modo, produtos, marcas, serviços e preços podem ser interpretados tanto como fontes de reforços quanto de eventos aversivos (OliveiraCastro et al., 2008).

Com base nesses conceitos, se torna possível interpretar, em termos comportamentais, parte dos fenômenos que a área de marketing denomina de diferenciação de marca como reforço informativo programado. Para identificar os níveis de reforço informativo das marcas, as alternativas de marcas ofertadas podem ser interpretadas como um conjunto de contingências sociais programadas, isto é, a compra e o uso de cada uma das marcas estariam associados a determinadas ações e reações das pessoas de determinados grupos (Foxall et al., 2004; Oliveira-Castro et al., 2008). O reforço informativo pode ser associado com diferenciação de marcas, pois as marcas mais conhecidas e promovidas são geralmente associadas com altos níveis de prestígio, status social e confiabilidade (Oliveira-Castro, Foxall, \& Schrezenmaier, 2005; Foxall et al., 2007). Assim, a compra de uma marca que é conhecida e de boa qualidade deve estar correlacionada com aumentos na probabilidade de reforço social por parte das pessoas do grupo, quando comparada com a compra de uma marca desconhecida ou considerada como possuindo baixa qualidade.

O nível de reforço informativo de marcas tem sido mensurado a partir de dados coletados por intermédio de questionários com perguntas que utilizam escalas de respostas tipo Likert de quatro pontos, usadas em questionários para mensurar e classificar atitudes, opiniões ou percepções das pessoas (cf. Allen \& Seaman, 2007). Tipicamente, pede-se ao consumidor para responder duas questões: a) A marca é conhecida? (0 - desconhecida; 1
- pouco conhecida; 2 - medianamente conhecida; e 3 - muito conhecida) e b) Qual o nível de qualidade da marca? (0 - desconhecida; 1 - baixa qualidade; 2 - média qualidade; e 3 - alta qualidade). A Média de Conhecimento e Qualidade (MCQ), determinada pela média aritmética dos dois valores $[\mathrm{MCQ}=$ (Conhecimento + Qualidade)/2] (Pohl \& OliveiraCastro, 2008; Nalini, Cardoso, \& Cunha, 2013), indica se as consequências programadas têm alto, médio ou baixo nível informativo (Oliveira-Castro et al., 2010). Assim, MCQ tem sido adotada como uma medida proximal do nível de reforço informativo, que possibilita sondar contingências sociais programadas, consistente com uma interpretação analítico-comportamental.

\section{Estudos anteriores sobre escolha e nível de reforço informativo de marcas}

Uncles, Ehrenberg e Hammond (1995) resumiram uma série de estudos sobre preferência de marcas que revelaram algumas características dos padrões de compra dos consumidores, os quais foram replicados para dezenas de categorias de produtos e serviços em diversos países. Dentre os principais achados, pode-se mencionar a constatação de que poucos consumidores são completamente leais a uma marca de produtos comprados frequentemente durante o período de um ano. Observou-se, também, que a maioria tem um repertório composto de um subconjunto de marcas oferecidas na categoria do produto que eles mais compram e as marcas que formam o repertório de um consumidor funcionam como substitutas.

Entre as pesquisas sobre escolha de marcas utilizando o BPM, Foxall et al. (2004) investigaram se os repertórios de marcas dos consumidores estavam relacionados aos níveis de reforço utilitário e informativo programados pelas marcas. Os produtos investigados incluíam produtos de compra rotineira de supermercados, entre eles feijão enlatado, biscoito, cereal, manteiga, queijo, suco de frutas, café solúvel, margarina e chá. Os autores utilizaram dados de painel que continham informações de compra de 80 consumidores durante 16 semanas. Os dados incluíam informações sobre cada compra realizada 
por cada consumidor durante o período, tais como a marca, tipo de produto, preço, número de unidades e peso da embalagem. Os autores classificaram cada marca conforme dois níveis de reforço utilitário (1 - produto simples e 2 - produto diferenciado) e três níveis de reforço informativo (1 - marcas populares, 2 - marcas sem diferenciação e 3 - marcas especializadas). Descobriram que a maioria dos indivíduos compra, predominantemente, marcas pertencentes ao mesmo nível de reforço, sugerindo que o repertório de marcas é formado com base no nível de reforçamento programado. Constataram, também, que padrões de comportamento podem ser determinados por combinações que evitam consequências aversivas, como pagar preço mais alto, e maximizam tanto o reforço informativo quanto o utilitário.

Oliveira-Castro et al. (2008) examinaram se a relação entre o valor da marca para o consumidor e o desempenho da marca depende da categoria do produto. $\mathrm{O}$ valor da marca, que se relaciona com o nível de diferenciação da marca, foi mensurado por intermédio de questionários que sondaram as contingências de reforço social para comprar cada marca, examinando quais seriam consideradas "conhecidas e de alta qualidade" pelo ambiente social (medida de MCQ). O desempenho da marca foi avaliado de acordo com a fatia de mercado e o seu preço médio. Foram investigadas 15 categorias de produtos de supermercado em dois países: café, margarina, sabão em pó, achocolatado, amaciante, detergente, feijão preto, milho, óleo, maionese e biscoito no Brasil; e feijão enlatado, biscoito, suco de frutas e gorduras amarelas no Reino Unido. Os resultados mostraram que a relação entre o valor da marca, medida pelo MCQ, e seu desempenho varia de acordo com a categoria de produto e sugeriram que não é rentável investir em diferenciação de marcas de categorias de produtos que se comportam como commodities. Além disso, níveis de reforço informativo e utilitário maiores - maior diferenciação da marca, estiveram associados a aumentos no faturamento. Os resultados também mostraram que é possível descobrir quanto a fatia de mercado pode aumentar com aumentos no MCQ, possibilitando avaliar o possível retorno do investimento na marca, e quanto a fatia de mercado pode diminuir com a elevação dos preços, o que pode auxiliar estratégias de precificação.
Oliveira-Castro et al. (2010) demonstraram a utilidade de integrar o BPM com a Lei de Igualação (Baum, 1974; Herrnstein, 1970) para explicar a escolha de marcas de produtos de compra rotineira, considerando as consequências quantitativas e qualitativas. Para isso, mostrou-se que os gastos com esses produtos mudam sistematicamente com promoções de preços, quantidade comprada, reforço utilitário e reforço informativo, em ordem decrescente de importância. Além disso, consumidores aumentam o montante gasto para obter quantidades maiores de dado produto e maior nível de reforço utilitário. No caso do reforço informativo, os efeitos sobre o gasto foram pequenos porque produtos de compra rotineira de supermercado constituem, tipicamente, um cenário com baixo nível desse reforço.

De maneira geral, esses trabalhos corroboram a tese de que o comportamento do consumidor é influenciado por contingências programadas de reforço social, denominadas de reforço informativo no BPM (Foxall, 1999). Além disso, ressaltam que a medida de MCQ pode ser adotada para sondar essas contingências, isto é, para mensurar o nível de reforço informativo programado associado a cada marca (Oliveira-Castro et al., 2008).

\section{Nível de reforço informativo de marcas e ponto de venda}

De acordo com a literatura na área de marketing (cf. Kotler \& Keller, 2012), uma das variáveis importantes do contexto de uma marca, que pode influenciar o comportamento do consumidor, é o ponto de venda, definido como o local em que bens ou serviços são vendidos ao consumidor final. O ponto de venda pode agrupar lojas e, com isso, aumentar o poder de atração sobre os clientes e oferecer a facilidade de encontrar vários produtos no mesmo lugar, como no caso de shoppings centers, o que os torna convenientes para os consumidores (Kotler \& Armstrong, 2007). Nos shoppings há lojas âncoras, que são conhecidas pelo público e são importantes porque aumentam a atração de consumidores e asseguram afluência e trânsito destes pelo ambiente (Glossário Abrasce, 2017). Segundo Parente e Kato (2001), a localização interfere no desempenho do 
estabelecimento, pois os consumidores que moram dentro de uma área geográfica relativamente pequena ao redor da loja - a área de influência, são responsáveis pela maioria das compras. Além disso, variáveis como acesso ao transporte, características do local, tamanho do estabelecimento, variedade, qualidade e preço interferem no tipo de consumidor que frequenta a loja.

O presente trabalho buscou verificar se essas variáveis, associadas aos shoppings, influenciam o nível de reforço informativo associado às marcas. Para isso, comparou o nível de reforço informativo de marcas de moda feminina entre grupos de pessoas que frequentam diferentes shoppings centers. Moda feminina é uma categoria de produto em que é perceptível a influência de fatores culturais e sociais que prevalecem no grupo que o indivíduo interage, pois o vestuário indica a situação pessoal do consumidor para todos que o observam, e por isso pode ser fonte relevante de reforços sociais. Essa importância social das vestimentas já era ressaltada na literatura econômica do Século XIX, ao afirmarem que o modo de se vestir evidencia o sucesso individual, o valor social, os gastos supérfluos e também a imagem de que produtos muito baratos são impróprios e inferiores (cf. Veblen, 1899, p. 124, 125 e 126).

Ao interpretar diferenciação de marcas como contingências programadas de reforço social, com base no BPM, espera-se que os níveis do reforço informativo variem a depender do ambiente social no qual a marca é comercializada. Essa predição difere de uma teoria de diferenciação de marcas que assume que o valor de uma marca é uma característica própria relativamente independente de seu ambiente (e.g., Aaker, 1997).

\section{Objetivos}

De acordo com o BPM, o reforço informativo é influenciado pelo grupo de referência do indivíduo e, por isso, pelo ambiente ou contexto. A medida MCQ, por sua vez, seria uma possibilidade de mensurar contingências sociais programadas. Tendo isso em vista, o principal objetivo da pesquisa foi examinar a relação entre a diferenciação das marcas e o ponto de venda - o ambiente. De modo mais específico, o objetivo foi verificar se o nível de reforço informativo de uma mesma marca pode ser diferente em shoppings qualitativamente distintos. Além disso, outro objetivo foi analisar a direção da relação, isto é, se um ambiente, com características que fazem com que seu nível informativo seja mais alto, eleva ou diminui o nível informativo da marca, e se isso depende das outras marcas que estão no mesmo ponto de venda (ambiente).

\section{Método}

\section{Participantes}

Participaram da pesquisa 120 indivíduos, moradores do Distrito Federal. A amostra foi composta $85,8 \%$ do gênero feminino. A faixa etária foi 33,3\% com idade entre 18 e 24 anos, $32,5 \%$ entre 25 e 34 anos, $20 \%$ entre 35 e 44 anos e o restante com idade maior que 45 anos. O nível de escolaridade foi $29,2 \%$ com pós-graduação completa e $27,5 \%$ incompleta ou cursando, $16,7 \%$ tinham superior completo enquanto $22,5 \%$ incompleto ou cursando, e $11,3 \%$ declararam ter ensino médio completo ou estarem cursando.

Aproximadamente $35 \%$ dos participantes eram estudantes de graduação ou pós, os demais tinham profissões diversas (psicólogo, professor, nutricionista, economista, administrador, bancário, advogado, agente de portaria e de viagem, arquiteto, servidor público, auxiliar de serviços gerais, entre outras). A renda pessoal foi de até um salário mínimo para $24,2 \%$ da amostra, de um a dois salários para $20 \%$, de dois a cinco salários para $21,7 \%$ e acima de cinco salários para $34,1 \%$. A renda familiar para $54,2 \%$ dos participantes era mais de 10 salários mínimos, para $20,8 \%$ de cinco a 10 salários, e $25 \%$ menos de cinco salários.

De todos os participantes, $75 \%$ disseram que costumam comprar moda feminina em shoppings. Disseram frequentar o Conjunto Nacional 60\% do total, o Park Shopping 68,3\% e o Iguatemi 35\%, alguns participantes declararam frequentar dois ou os três shoppings alvo. Quanto a regularidade, 15\% dos indivíduos declararam frequentar shoppings até uma vez por semana no mínimo, 50\% a cada 15 dias ou uma vez por mês no mínimo, $25 \%$ uma vez a cada três meses aproximadamente e o restante quase não vai ao shopping. 


\section{Instrumento e Procedimentos Analíticos}

Questionários são instrumentos bastante utilizados nas ciências sociais empíricas para compreender o comportamento humano e têm a vantagem de permitir a generalização (Günther, 2008). Além disso, para os objetivos do trabalho, sua aplicação possibilita investigar as contingências sociais existentes, no sentido de que as marcas que os consumidores consideram muito conhecidas e de alta qualidade são as mais suscetíveis de gerarem aprovação social para quem compra (Oliveira-Castro, Cavalcanti, \& Foxall, 2015).

Assim, foi aplicado um questionário para sondar o reforço informativo das marcas e para reunir dados sociodemográficos. Foram levantadas informações sobre o nível de conhecimento (0 - desconhecida, 1 - pouco, 2 - medianamente e 3 - muito conhecida) e de qualidade ( 0 - desconhecida/sem opinião, 1 - baixa, 2 - média e 3 - alta qualidade) para calcular a medida MCQ, isto é, a média aritmética da soma da média de conhecimento e da média de qualidade para cada marca $[\mathrm{MCQ}=($ Média de Conhecimento + Média de Qualidade)/2]. Valores entre 2 e 3 indicam que as consequências produzidas têm alto nível informativo, entre 1 e 2 médio nível, e entre 0 e 1 têm baixo nível informativo. Além disso, foi questionado: Você usaria? (0 - de forma alguma, 1 - pouco provavelmente, 2 - provavelmente, 3 - muito provavelmente); Você compraria? (0 - de forma alguma, 1 - pouco provavelmente, 2 - provavelmente, 3 - muito provavelmente); $\mathrm{O}$ preço é justo considerando a qualidade da marca? (0 - sem opinião, 1 - de forma alguma, 2 - parcialmente justo, 3 - justo). Para dados sociodemográficos foram abordadas questões referentes à idade, ocupação, renda média pessoal e familiar, escolaridade, quais lojas e shoppings frequenta e de quanto em quanto tempo faz compras e vai ao shopping.

As 13 marcas que compuseram o questionário (Any Any, Arezzo, Dudalina, Hering, Jogê, Luigi Bertolli, M. Officer, Mr. Foot, Nike, Puket, Santa Lolla, Scala e Zara) são de moda feminina e possuíam lojas em três shoppings centers em Brasília (Conjunto Nacional, Park Shopping e Iguatemi). Algumas lojas âncoras fizeram parte do questionário (C\&A, Marisa, Renner e Riachuelo), bem como alguns hipermercados (Carrefour, Extra e Walmart), apesar de não serem comuns aos três shoppings. No entanto, foram excluídos das análises porque não houve diferença significativa nos resultados e não são a marca em si, mas lojas que ofertam várias marcas.

Os shoppings foram escolhidos porque, além de disporem do maior número de lojas em comum, apresentam diferenças significativas na localização e no perfil dos frequentadores. Considerando as variáveis propostas por Parente e Kato (2001), o Conjunto Nacional está na região central e as pessoas têm fácil acesso ao transporte público nas proximidades. O Park Shopping, apesar de estar mais afastado, também tem mais de uma opção de transporte, porém se diferencia do Conjunto por concentrar maior variedade de lojas. O Iguatemi está localizado num lugar distante e de difícil acesso por meio do transporte público, além de poder ser considerado de mais alto padrão socioeconômico por abrigar lojas de luxo exclusivas como Christian Louboutin, Dolce \& Gabbana, Gucci, Louis Vuitton e Prada.

Em resumo, a diferenciação da marca foi associada ao nível de reforço informativo de marcas de moda feminina - mensurado pelo MCQ, e os pontos de vendas comparados foram shoppings centers qualitativamente diferentes entre si e frequentados por grupos distintos, mas com marcas comuns.

\section{Resultados}

Para examinar se os shoppings são estatisticamente diferentes entre si, foi feito o teste não-paramétrico Kruskal-Wallis entre cada shopping e a renda familiar dos participantes. Esse teste permitiu comparar a renda familiar dos indivíduos que frequentam cada um dos três estabelecimentos com a renda familiar dos indivíduos que não frequentam. O teste mostrou que existe efeito da renda sobre os shoppings Conjunto Nacional $\left[\chi^{2}(3)=13,149\right.$, $\mathrm{p}<0,01]$ e Iguatemi $\left[\chi^{2}(3)=16,109, p<0,01\right]$. Logo, em média ranqueada, a renda dos frequentadores do Conjunto Nacional é diferente da renda dos não frequentadores, o mesmo ocorre com a renda dos indivíduos que frequentam o Iguatemi ou não. No caso do Park Shopping, o teste não foi significativo $\left[\chi^{2}(3)=3,777, p>0,05\right]$, indicando que a renda familiar dos participantes que frequentam não difere da 
renda dos que não frequentam esse shopping.

Como a maioria dos participantes respondeu que vai em mais de um dos três shoppings investigados, estes foram transformados em três variáveis dicotômicas $(0,1)$ : um se frequenta o Conjunto Nacional e zero se não frequenta; um se frequenta o Park Shopping e zero se não frequenta; e um se frequenta o Iguatemi e zero se não frequenta. As respostas de seis participantes que não frequentam pelo menos um dos shoppings selecionados foram consideradas missings.
A medida MCQ foi calculada a partir das médias de conhecimento e qualidade para cada marca em cada shopping, conforme Oliveira-Castro et al. (2008): primeiro foi calculada a média de conhecimento e a média de qualidade considerando todos os participantes, para depois calcular a média de conhecimento e qualidade para cada marca em cada shopping. A marca com maior média foi a Nike e com menor média foi a Any Any para os três shoppings, conforme apresentado na Tabela 1.

Tabela 1. Média de Conhecimento e Qualidade (MCQ) das marcas para cada shopping

\begin{tabular}{|c|c|c|c|c|c|c|}
\hline Marcas & $\begin{array}{l}\text { MCQ para fre- } \\
\text { quentadores do } \\
\text { Conjunto Nacional }\end{array}$ & $\begin{array}{l}\text { MCQ para não } \\
\text { frequentadores } \\
\text { do Conjunto } \\
\text { Nacional }\end{array}$ & $\begin{array}{l}\text { MCQ para fre- } \\
\text { quentadores do } \\
\text { Park Shopping }\end{array}$ & $\begin{array}{l}\text { MCQ para não } \\
\text { frequentadores } \\
\text { do Park Shopping }\end{array}$ & $\begin{array}{l}\text { MCQ para fre- } \\
\text { quentadores } \\
\text { do lguatemi }\end{array}$ & $\begin{array}{l}\text { MCQ para } \\
\text { não frequen- } \\
\text { tadores do } \\
\text { Iguatemi }\end{array}$ \\
\hline Any Any & 1,23 & 1,92 & 1,63 & 1,09 & 1,85 & 1,27 \\
\hline Arezzo & 2,47 & 2,70 & 2,61 & 2,42 & 2,63 & 2,51 \\
\hline Dudalina & 1,88 & 2,32 & 2,17 & 1,72 & 2,23 & 1,94 \\
\hline Hering & 2,55 & 2,44 & 2,50 & 2,53 & 2,49 & 2,52 \\
\hline Jogê & 1,44 & 1,90 & 1,79 & 1,14 & 2,07 & 1,34 \\
\hline $\begin{array}{l}\text { Luigi } \\
\text { Bertolli }\end{array}$ & 1,67 & 1,92 & 1,82 & 1,61 & 2,11 & 1,56 \\
\hline M. Officer & 2,11 & 2,29 & 2,30 & 1,84 & 2,37 & 2,06 \\
\hline Mr. Foot & 2,02 & 2,18 & 2,23 & 1,69 & 2,17 & 2,03 \\
\hline Nike & 2,82 & 2,87 & 2,84 & 2,83 & 2,94 & 2,78 \\
\hline Puket & 1,99 & 2,14 & 2,15 & 1,77 & 2,23 & 1,94 \\
\hline $\begin{array}{l}\text { Santa } \\
\text { Lolla }\end{array}$ & 1,40 & 1,89 & 1,70 & 1,27 & 1,95 & 1,36 \\
\hline Scala & 1,58 & 1,86 & 1,80 & 1,36 & 1,90 & 1,55 \\
\hline Zara & 2,35 & 2,48 & 2,39 & 2,41 & 2,52 & 2,32 \\
\hline
\end{tabular}

Nota. $\mathrm{N}=114$

Para averiguar se o shopping frequentado é capaz de prever diferentes medidas de MCQ por participante, foram realizadas análises com regressões lineares múltiplas para todas as marcas. As premissas de tamanho da amostra, valores independentes e relação linear entre as variáveis foram atendidas. As premissas de resíduos independentes, ausência de outliers, homocedasticidade, normalidade dos resíduos e ausência de multicolinearidade foram testadas e também consideradas adequadas (cf. Field, 2009).
$\mathrm{Na}$ primeira regressão, colocou-se a medida MCQ das marcas como variável de saída (dependente) e os shoppings frequentados pelos participantes da pesquisa como variáveis preditoras (independentes). A análise resultou em um modelo estatisticamente significativo $[\mathrm{F}(3,110)=7,503$; $\left.\mathrm{p}<0,01 ; \mathrm{R}^{2}=0,170\right]$ e mostrou que o Park Shopping e o Iguatemi são preditores da medida MCQ. A equação que descreve essa relação é:

$$
\mathrm{MCQ}=\beta_{1}+\beta_{2} \mathrm{C}+\beta_{3} \mathrm{P}+\beta_{4} \mathrm{I}+\varepsilon
$$


Em que o $M C Q$ é o nível de reforço informativo associado a diferenciação da marca, $C$ é o shopping Conjunto Nacional, $P$ é o Park Shopping, I é o Iguatemi, $\beta 1$ a $\beta 4$ são parâmetros obtidos empiricamente e $\varepsilon$ é uma medida de erro. Pode-se observar na Tabela 2, que para essa primeira regressão, os parâmetros foram significantes e positivos para o Park Shopping e o Iguatemi, indicando que o nível do MCQ de uma marca tendeu a aumentar quando ela é avaliada por frequentadores desses shoppings. O parâmetro para o Conjunto Nacional não foi significativo e apresentou coeficiente negativo.

Tabela 2. Coeficientes das regressões com as VD's MCQ, Conhecimento e Qualidade

\begin{tabular}{|c|c|c|c|c|c|c|c|c|c|}
\hline \multirow[t]{2}{*}{ VI's/VD's } & \multicolumn{3}{|l|}{$\mathrm{MCQ}$} & \multicolumn{3}{|c|}{ Conhecimento } & \multicolumn{3}{|c|}{ Qualidade } \\
\hline & $B$ & Beta & $t$ & $B$ & Beta & $t$ & $B$ & Beta & $t$ \\
\hline Constante & $1,727^{\star *}$ & & 11,519 & $1,765^{\star *}$ & & 12,199 & $1,689^{\star *}$ & & 9,310 \\
\hline Conjunto Nacional & $-0,087$ & $-0,073$ & $-0,772$ & $-0,016$ & $-0,014$ & $-0,150$ & $-0,157$ & $-0,112$ & $-1,157$ \\
\hline Park Shopping & $0,359^{* *}$ & 0,282 & 3,118 & $0,396^{* *}$ & 0,321 & 3,564 & $0,322^{*}$ & 0,214 & 2,311 \\
\hline Iguatemi & $0,345^{\star *}$ & 0,291 & 3,100 & $0,359^{\star \star}$ & 0,312 & 3,346 & $0,330 *$ & 0,236 & 2,456 \\
\hline
\end{tabular}

Nota. $\mathrm{N}=114 ;{ }^{*} \mathrm{p}<0,05 ;{ }^{* *} \mathrm{p}<0,01$

Na segunda regressão, a variável de saída foi apenas o nível de conhecimento das marcas, um dos fatores que compõem a medida MCQ, com intuito de perscrutar se marcas muito conhecidas com nível de qualidade baixo ou marcas menos conhecidas com nível de qualidade maior poderiam enviesar os resultados. O modelo foi estatisticamente significativo $\left[F(3,110)=7,995 ; \mathrm{p}<0,01 ; \mathrm{R}^{2}=0,179\right]$. A equação que descreve essa relação é:

$$
\mathrm{NC}=\beta_{1}+\beta_{2} \mathrm{C}+\beta_{3} \mathrm{P}+\beta_{4} \mathrm{I}+\varepsilon
$$

Em que o NC é o nível médio de conhecimento das 13 marcas, $C$ é o shopping Conjunto Nacional, $P$ é o Park Shopping, $I$ é o Iguatemi, $\beta 1$ a $\beta 4$ são parâmetros obtidos empiricamente e $\varepsilon$ é uma medida de erro. Pode-se observar também na Tabela 2, que para essa regressão, os parâmetros foram significantes e positivos para o Park Shopping e o Iguatemi, indicando que o nível do conhecimento de uma marca tendeu a aumentar quando ela é avaliada por frequentadores desses shoppings. O parâmetro para o Conjunto Nacional não foi significativo e apresentou coeficiente negativo. Os resultados da segunda regressão corroboram os da primeira.

Pelo mesmo motivo da segunda, na terceira regressão a variável dependente foi a média do nível de qualidade. Nesse caso o modelo também foi estatisticamente significativo $[\mathrm{F}(3,110)=5,364$; $\left.\mathrm{p}<0,01 ; \mathrm{R}^{2}=0,128\right]$. A equação que descreve essa relação é:

$$
\mathrm{NQ}=\beta_{1}+\beta_{2} \mathrm{C}+\beta_{3} \mathrm{P}+\beta_{4} \mathrm{I}+\varepsilon
$$

Em que o NQ é o nível médio de qualidade das marcas, $C$ é o shopping Conjunto Nacional, $P$ é o Park Shopping, I é o Iguatemi, $\beta 1$ a $\beta 4$ são parâmetros obtidos empiricamente e $\varepsilon$ é uma medida de erro. Para essa regressão, os parâmetros foram significantes e positivos também para o Park Shopping e para o Iguatemi, indicando que o nível de qualidade de uma marca tendeu a aumentar quando é avaliada por frequentadores desses shoppings. O parâmetro para o Conjunto Nacional não foi significativo e apresentou coeficiente negativo. Os coeficientes dessa regressão são apresentados na Tabela 2 .

A regressão linear múltipla também foi utilizada para verificar se, além do shopping frequentado, dados sociodemográficos como a renda familiar, o gênero, a faixa etária e a escolaridade são capazes de prever a média de MCQ por participante para todas as marcas. A análise resultou em um modelo estatisticamente significativo $\left[F(7,106)=8,590 ; p<0,01 ; R^{2}=0,362\right]$, em que o Park Shopping, o Iguatemi e o gênero são preditores da medida MCQ, conforme mostra a Tabela 3. 
Tabela 3. Coeficientes da regressão com dados sociodemográficos

\begin{tabular}{|c|c|c|c|}
\hline \multirow[t]{2}{*}{ VI's/VD's } & \multicolumn{3}{|l|}{$\mathrm{MCQ}$} \\
\hline & $B$ & Beta & $t$ \\
\hline Constante & $1,549^{\star *}$ & & 6,364 \\
\hline Conjunto Nacional & $-0,012$ & $-0,010$ & $-0,118$ \\
\hline Park Shopping & $0,277^{\star \star}$ & 0,217 & 2,538 \\
\hline Iguatemi & $0,383^{\star *}$ & 0,323 & 3,614 \\
\hline Renda Familiar & 0,084 & 0,143 & 1,552 \\
\hline Gênero & $-0,722^{\star *}$ & $-0,427$ & $-5,304$ \\
\hline Faixa Etária & 0,087 & 0,180 & 1,939 \\
\hline Escolaridade & $-0,029$ & $-0,061$ & $-0,619$ \\
\hline
\end{tabular}

Nota. $\mathrm{N}=114 ; * * \mathrm{p}<0,01$

Tabela 4. Coeficientes da regressão com disposições dos consumidores

\begin{tabular}{|c|c|c|c|}
\hline \multirow[t]{2}{*}{ VI's/VD's } & \multicolumn{3}{|l|}{$\mathrm{MCO}$} \\
\hline & $B$ & Beta & $t$ \\
\hline Constante & $0,492^{\star \star}$ & & 3,123 \\
\hline Conjunto Nacional & $-0,067$ & $-0,057$ & $-0,901$ \\
\hline Park Shopping & $0,190^{*}$ & 0,149 & 2,277 \\
\hline Iguatemi & $0,203^{\star *}$ & 0,171 & 2,629 \\
\hline Usaria & $0,444^{\star \star}$ & 0,456 & 4,381 \\
\hline Compraria & $-0,081$ & $-0,088$ & $-0,851$ \\
\hline Preço Justo & $0,371^{\star \star}$ & 0,418 & 5,360 \\
\hline
\end{tabular}

Nota. $\mathrm{N}=114 ;{ }^{\star} \mathrm{p}<0,05 ;{ }^{\star *} \mathrm{p}<0,01$

A equação que descreve essa relação é:

$$
\begin{aligned}
& \mathrm{MCQ}=\beta_{1}+\beta_{2} \mathrm{C}+\beta_{3} \mathrm{P}+\beta_{4} \mathrm{I}+\beta_{5} \mathrm{R}+\beta_{6} \mathrm{G}+\beta_{7} \\
& \mathrm{~F}+\beta_{8} \mathrm{E}+\varepsilon \quad \text { (4) }
\end{aligned}
$$

Em que o MCQ é o nível de reforço informativo associado a diferenciação da marca, $C$ é o shopping Conjunto Nacional, $P$ é o Park Shopping, $I$ é o Iguatemi, $R$ é a renda familiar, $G$ é o gênero, $F$ é a faixa etária e $E$ é a escolaridade dos consumidores entrevistados. Os parâmetros continuaram sendo significantes e positivos para o Park Shopping e o Iguatemi. Já o parâmetro do gênero, variável dicotômica com valor zero para feminino e um para masculino, foi negativo e significante, indicando que o nível do MCQ de uma marca tendeu a diminuir quando foi avaliada por participantes do gênero masculino.
Por fim, a regressão linear múltipla foi utilizada para verificar se, além do shopping frequentado, o fato do participante dizer se usaria, se compraria e se acha o preço justo das marcas são capazes de prever a medida MCQ por participante para todas as marcas. A análise resultou em um modelo estatisticamente significativo $[\mathrm{F}(6,107)=33,350 ; \mathrm{p}<0,01$; $\left.\mathrm{R}^{2}=0,652\right]$.

A equação que descreve essa relação é:

$$
\begin{aligned}
& \mathrm{MCQ}=\beta_{1}+\beta_{2} \mathrm{C}+\beta_{3} \mathrm{P}+\beta_{4} \mathrm{I}+\beta_{5} \mathrm{U}+\beta_{6} \mathrm{~B}+ \\
& \beta_{7} \mathrm{P}_{j}+\varepsilon \quad(5)
\end{aligned}
$$

Em que o MCQ é o nível de reforço informativo associado a diferenciação da marca, $C$ é o shopping Conjunto Nacional, $P$ é o Park Shopping, $I$ é o Iguatemi, $U$ é se usaria, $B$ se compraria e $P j$ se acha o preço justo. Os parâmetros foram significantes e 
positivos para o Park Shopping, o Iguatemi, se usaria e se acha o preço justo, indicando que o nível do MCQ de uma marca tendeu a aumentar quando é avaliada por frequentadores desses shoppings, quando existe a possibilidade de usar e quando os participantes achavam o preço justo, conforme apresentado na Tabela 4.

\section{Discussão}

Os objetivos da presente pesquisa foram: examinar a relação entre a diferenciação das marcas e o ponto de venda, especificamente, verificar se o nível de reforço informativo de uma mesma marca pode ser diferente em shoppings qualitativamente distintos; $\mathrm{e}$ analisar a direção dessa relação, ao verificar se um ambiente com nível informativo mais alto eleva ou diminui o nível informativo da marca e se isso depende das outras marcas que estão próximas ao seu ponto de venda.

Com relação ao primeiro objetivo, observou-se que a diferenciação da marca está relacionada com o ponto de venda e que uma mesma marca sinaliza nível de reforço informativo diferente em shoppings qualitativamente distintos. Uma relação empírica importante foi que shoppings centers mais diferenciados, por abrigarem marcas com maior nível informativo, como marcas de luxo, e por terem localização e características distintas (cf. Parente e Kato, 2001), influenciam na avaliação do nível de reforço social dessas marcas (cf. Oliveira-Castro et al., 2015). Isso difere da tese de que o valor de uma marca é uma característica própria relativamente independente de seu ambiente (cf. Aaker, 1997).

É importante destacar que a diferença qualitativa entre os shoppings foi testada, e foi comprovado, estatisticamente, que o Iguatemi é um shopping cujos frequentadores possuem nível socioeconômico mais elevado. Se o indivíduo frequenta o Park Shopping ou o Iguatemi, ou ambos, o MCQ aumenta, em média. O Conjunto Nacional não mostrou relação significativa com o MCQ, isso sugere que é um ambiente com nível de reforço social mais baixo, que não prevê a diferenciação de marcas quando é avaliada por seus frequentadores. Esse achado nos permite afirmar que a diferenciação está relacionada ao grupo que frequenta o local e ao nível socioeconômico dos indivíduos. Isso corrobora a tese de que o cenário de consumo, no qual o consumidor é exposto a eventos, funciona como estímulo discriminativo e sinaliza as consequências para as possíveis escolhas (cf. Oliveira-Castro \& Foxall, 2015; Oliveira-Castro et al., 2008).

Ainda considerando os estímulos antecedentes ao comportamento do consumidor, o fato do gênero poder ser considerado um preditor da medida MCQ indica que a avaliação das marcas feita pelas mulheres é diferente da dos homens. Observouse que as mulheres valorizam mais as marcas de moda feminina do que os homens, como é de se esperar, pois o gênero pode ser interpretado como uma variável antecedente, de acordo com o BPM, que remete à história individual. Isto é, o gênero é uma proxy da história de aprendizagem que prevê o reforço social e, consequentemente, está associado a diferenciação das marcas (cf. Oliveira-Castro \& Foxall, 2015; Oliveira-Castro et al., 2008). Ressaltase que isso não significa, necessariamente, que homens não valorizam marcas, mas que por se tratar de marcas de moda feminina, mesmo que algumas ofertem produtos masculinos, os homens podem não conhecê-las.

Quanto às disposições dos consumidores de usar, comprar e achar o preço das marcas justo, descobriu-se que os participantes que usariam a marca e que acham o seu preço justo a valorizam mais do que quem não usaria e não acha o preço adequado, conforme esperado. Esses resultados sugerem correlações entre o MCQ e as verbalizações dos respondentes relacionadas a uso e compra, conforme tem sido ressaltado pela literatura que investiga valor de marca (cf. Oliveira-Castro et al., 2008). A disposição de usar e de achar o preço justo pode ser interpretada como decorrente da história de aprendizagem individual, isto é, deriva da experiência que o indivíduo teve anteriormente com a marca ou do que ouviu falar no grupo de referência.

É possível que a medida MCQ, o nível de conhecimento e o nível de qualidade de algumas marcas tenham sido mais baixos devido às características específicas da amostra, como ocupação e renda pessoal média. Essas características podem ter contribuído para o desconhecimento de algumas marcas de moda feminina, justamente por poder ser considerada uma categoria com mais alto 
nível de reforço informativo quando comparada com outras categorias de produtos. Isto é, moda feminina constitui uma categoria de produto em que as escolhas dos consumidores são mais influenciadas por reforços sociais quando comparada com produtos de compra rotineira em supermercados, por exemplo. Esses estão em um cenário de consumo com baixo nível de reforço informativo tipicamente (cf. Oliveira-Castro et al., 2010). Isso está de acordo com a tese de que o nível de análise do reforço informativo e a relação do valor da marca e seu desempenho dependem da categoria do produto e do interesse da pesquisa (cf. Oliveira-Castro et al., 2008).

No que se refere à análise da direção da relação, isto é, se um ambiente com nível informativo mais alto eleva ou diminui o nível informativo da marca, descobriu-se que um shopping com nível informativo maior aumenta os potenciais reforços sociais de determinada marca. De acordo com os resultados obtidos nas regressões, a medida MCQ por participante para as marcas aumenta quando os consumidores são frequentadores do Park Shopping e do Iguatemi. Ao considerar que a compra e o uso de determinada marca estariam associados a determinadas ações e reações das pessoas de determinados grupos (cf. Foxall et al., 2004; Oliveira-Castro et al., 2008), pode-se afirmar que o ambiente, que é frequentado por grupos diferentes, também é influenciado pelas ações e reações das pessoas desses grupos.

Em resumo, o ponto de venda ser uma variável que influencia a diferenciação da marca corrobora a tese de que tal diferenciação depende do contexto no qual a marca se insere, como previsto pelo BPM (cf. Oliveira-Castro et al., 2008) e que MCQ é uma medida útil para mensurar reforços informativos como contingências sociais programadas. Além disso, o achado de que um ponto de venda, como um shopping, com nível informativo maior aumenta os potenciais reforços sociais das marcas ali comercializadas não é trivial. $O$ efeito poderia ter sido inverso, pois poder-se-ia pensar que o nível de diferenciação de uma marca mediana, por exemplo, fosse influenciado negativamente por estar próxima de marcas mais diferenciadas. Os resultados indicam um efeito de assimilação, não de contraste (cf. Shimp, Stuart, \& Engle, 1991), entre contexto e diferenciação de marcas, isso significa que um contexto muito diferenciado tenderia a aumentar o nível de diferenciação de uma marca, quando comparado com um contexto pouco diferenciado.

\section{Considerações finais}

Ao utilizar o arcabouço teórico do BPM para analisar diferenciação de marcas como contingências sociais programadas, observou-se que a mesma marca sinaliza níveis de reforços informativos diferentes, dependendo do ponto de venda, e que um shopping com nível informativo maior aumenta os potenciais reforços sociais associados a determinada marca. A medida MCQ depende do nível de diferenciação das demais marcas presentes no shopping, ou seja, depende do ambiente.

Além disso, o trabalho demonstra a utilidade da Teoria Analítico-Comportamental para examinar comportamentos de escolha de marcas, ao utilizar o MCQ como medida proximal para mensurar reforços informativos como contingências sociais programadas. Pode-se afirmar, assim, que a presente pesquisa é um exemplo de como a Análise do Comportamento permite sondar contingências sociais programadas. Para estudos futuros, sugere-se uma amostra com mais participantes e variação das marcas incluindo um número maior e mais diverso, inclusive marcas de luxo e de moda masculina. Além disso, sugere-se investigar outras categorias de produtos e que estejam em um cenário de consumo diferente, isto é, diferentes contextos de venda, como exemplo, comparar shoppings com feiras e aeroportos. Essa investigação poderia ter implicações práticas na criação de estratégias de posicionamento relacionadas à distribuição da marca, ou seja, onde a loja deveria ser instalada para maximizar o seu valor para os consumidores.

\section{Referências}

Aaker, J. L. (1997). Dimensions of brand personality. Journal of marketing research, 34(3), 347356. doi: 10.2139/ssrn. 945432

Allen, I. E., \& Seaman, C. A. (2007). Likert scales and data analyses. Quality progress, 40(7), 64. Recuperado de https://bit.ly/351pa89 
Austin, J. R., Siguaw, J. A., \& Mattila, A. S. (2003). A re-examination of the generalizability of the Aaker brand personality measurement framework. Journal of Strategic Marketing, 11(2), 7792. doi: 10.1080/0965254032000104469

Baum, W. M. (1974). On two types of deviation from the matching law: bias and undermatching. Journal of the Experimental Analysis of Behavior, 22(1), 231-242. doi: 10.1901/ jeab.1974.22-231

Baum, W. M. (2006). Compreender o behaviorismo: comportamento, cultura e evolução. Porto Alegre: Artmed Editora.

Doyle, S. A., \& Moore, C. M. (2018). Luxury Brand Outsiders: Understanding the Success of British and American Luxury Brands. In Global Luxury (pp. 65-86). Palgrave, Singapore.

Field, A. (2009). Discovering statistics using SPSS. London: SAGE.

Foxall, G. (1990). Consumer psychology in behavioral perspective. London and New York: Routledge.

Foxall, G. (1997). Marketing psychology: The paradigm in the wings. Springer.

Foxall, G. R. (1998). Radical behaviorist interpretation: generating and evaluating an account of consumer behavior. The Behavior Analyst, 21, 321-354. doi: 10.1007/bf03391971

Foxall, G. R. (1999). The substitutability of brands. Managerial and Decision Economics, 20, 241-257. doi: 10.1002/ (SICI) 1099-1468(199908)20:5<241::AID MDE936>3.0.CO;2-U

Foxall, G. R. (2010). Theoretical and Conceptual Advances in Consumer Behavior Analysis Invitation to Consumer Behavior Analysis. Journal of Organizational Behavior Management, 30, 92-109. doi: 10.1080/01608061003756307

Foxall, G. R. (2017). Advanced Introduction to Consumer Behavior Analysis. Glos: Elgar.

Foxall, G. R., Oliveira-Castro, J. M., \& Schrezenmaier, T. C. (2004). The behavioral economics of consumer brand choice: Patterns of reinforcement and utility maximization. Behavioral Processes, 66 (3), 235-260. doi: 10.1016/j.beproc.2004.03.007

Foxall, G. R., Oliveira-Castro, J. M., Schrezenmaier, T. C., \& James, V. K. (2007). The behavioral economics of brand choice. New York: Palgrave Macmillan.

Glossário Abrasce (2017). Glossário Definições e Convenções do setor de Shopping Centers. In Abrasce - Associação Brasileira de Shopping Centers. Acesso em 18/07/2017, https://bit. ly/2AIDnc0

Günther, H. (2008). Como elaborar um questionário. In: Pinheiro, J. Q. (Org.), Métodos de pesquisa nos estudos pessoa-ambiente (pp. 105147). São Paulo: Casa do Psicólogo.

Herrnstein, R. J. (1970). On the law of effect. Journal of the Experimental Analysis of Behavior, 13(2), 243-266. doi: 10.1901/jeab.1970.13-243

Keller, K. L., \& Lehmann, D. R. (2006). Brands and branding: Research findings and future priorities. Marketing science, 25(6), 740-759. doi: $10.1287 / \mathrm{mksc} .1050 .0153$

Kotler, P. \& Armstrong, G. (2007). Princípios de Marketing. São Paulo: Pearson Prentice Hall.

Kotler, P. \& Keller, K. L. (2012). Administração de Marketing. São Paulo: Pearson Education do Brasil.

Nalini, L. E. G., Cardoso, M. De M., \& Cunha, S. R. (2013). Comportamento do Consumidor: uma introdução ao Behavioral Perspective Model (BPM). Fragmentos de Cultura, 23(4), 489-505. Recuperado de https://bit.ly/31Jyprt

Oliveira-Castro, J. M., Foxall, G. R., \& Schrezenmaier, T. C. (2005). Patterns of consumer response to retail price differentials. Service Industries Journal, 25(3), 1-27. doi: 10.1080/02642060500050392

Oliveira-Castro, J. M., Foxall, G. R., \& Wells, V. K. (2010). Consumer Brand Choice: Money Allocation as a Function of Brand Reinforcing Attributes. Journal of Organizations Behavior Management, 30, 161-175. doi: 10.1080/01608061003756455

Oliveira-Castro, J. M., Foxall, G. R., James, V. K., Pohl, R. H. B. F., Dias, M. B., \& Chang, S. W. (2008). Consumer-based brand equity and brand performance. The Service Industries Journal, 28, 445-461. doi: $10.1080 / 02642060801917554$

Oliveira-Castro, J. M., \& Foxall, G. R. (2015). Dimensions of demand elasticity. In: Foxall, G. R., The Routledge Companion to Consumer 
Behavior Analysis (pp. 121-137). New York: Routledge Taylor \& Francis Group.

Oliveira-Castro, J. M., Cavalcanti, P. R. \& Foxall, G. R. (2015). What do consumers maximize? The analysis of utility functions in light of the Behavioral Perspective Model. In: Foxall, G. R. The Routledge Companion to Consumer Behavior Analysis (pp. 202-212). New York: Routledge Taylor \& Francis Group.

Parente, J. \& Kato, H. T. (2001). Área de influência: um estudo no varejo de supermercados. Revista de Administração de empresas, 41(2), 46-53. Recuperado de https://bit.ly/2oaQfFl

Pohl, R. H. B. F. \& Oliveira-Castro, J. M. (2008). Efeitos do nível de benefício informativo das marcas sobre a duração do comportamento de procura. RAC-Electronica, 2(3), 449-469. Recuperado de https://bit.ly/2MaiCLP

Shimp, T. A., Stuart, E. W., \& Engle, R. W. (1991). A program of classical conditioning experiments testing variations in the conditioned stimulus and context. Journal of Consumer Research, 18(1), 1-12. doi: 10.1086/209236

Skinner, B. F. (1953). Science and Human Behavior. New York: Macmillan.

Skinner, B. F. (1957). Verbal Behavior. Englewood Cliffs, New Jersey: Prentice-Hall.

Smith, W. R. (1956). Product differentiation and market segmentation as alternative marketing strategies. Journal of Marketing, 21, 3-8. Recuperado de https://bit.ly/2OhXt5c

Uncles, M., Ehrenberg, A., \& Hammond, K. (1995). Patterns of buyer behavior: Regularities, models, and extensions. Marketing Science, 14(3), 71-78. Recuperado de 10.1287/mksc.14.3.G71

Veblen, T. (1899). The theory of the leisure class. London: Unwin Books.

Vyncke, P. (2002). Lifestyle segmentation: From attitudes, interests and opinions, to values, aesthetic styles, life visions and media preferences. European journal of communication, 17(4), 445463. doi: $10.1177 / 02673231020170040301$

Wedel, M., \& Kamakura, W. A. (2012). Market segmentation: Conceptual and methodological foundations (Vol. 8). Springer Science \& Business Media.

Wells, V. K., Chang, S. W., Oliveira-Castro, J., \& Pallister, J. (2010). Market segmentation from a behavioral perspective. Journal of Organizational Behavior Management, 30(2), 176-198. doi: 10.1080/01608061003756505

\section{Informações do Artigo}

Histórico do artigo:

Submetido em: 14/10/2019

Primeira decisão editorial: 21/07/2020

Aceito em: 29/07/2020 